\title{
High frequency ultrasound with color Doppler in dermatology*
}

\author{
Elisa de Oliveira Barcaui ${ }^{1}$ \\ Flavia Paiva Proença Lobo Lopes ${ }^{1}$ \\ Carlos Baptista Barcaui
}

\author{
Antonio Carlos Pires Carvalho ${ }^{1}$ \\ Juan Piñeiro-Maceira²
}

DOI: http:/ / dx.doi.org/10.1590/abd1806-4841.20164446

\begin{abstract}
Ultrasonography is a method of imaging that classically is used in dermatology to study changes in the hypoderma, as nodules and infectious and inflammatory processes. The introduction of high frequency and resolution equipments enabled the observation of superficial structures, allowing differentiation between skin layers and providing details for the analysis of the skin and its appendages. This paper aims to review the basic principles of high frequency ultrasound and its applications in different areas of dermatology.
\end{abstract}

Keywords: Dermatology; Skin; Skin Neoplasms; Ultrasonography

\section{INTRODUCTION}

Diagnostic imaging methods are increasingly used in dermatology. ${ }^{1}$ Techniques such as dermoscopy, confocal microscopy, optical coherence tomography and high-frequency ultrasound (HFUS) enable the study of skin lesions in real time, non-invasively and without radiation. ${ }^{2-7}$ However, these tests vary considerably when evaluating its penetration, resolution and applicability. ${ }^{8}$

Ultrasonography with frequency higher than $7 \mathrm{MHz}$ allows the observation of the skin and its appendages, subcutaneous tissue and deep structures (muscles, tendons, bone margins and regional lymph nodes). Blood flow can be estimated with color Doppler use. ${ }^{9}$ The development of devices with frequencies above $15 \mathrm{MHz}$ allowed the differentiation of skin layers, increasing considerably its use in dermatology..$^{10}$ The high frequency equipment $(>15 \mathrm{~Hz})$ have little penetration and excellent resolution for the study of superficial structures. ${ }^{11}$ By analyzing the skin surface in three dimensions, the HFUS, associated with color Doppler, allows the investigation of tumor and inflammatory diseases, the measurement of skin thickness and evaluation of proposed treatments. ${ }^{12}$
In this article we will discuss the principles of the HFUS and the color Doppler, the technique for skin ultrasonography, the ultrasonographic features of normal skin and the main indications of this method of diagnostic imaging. This technique can be used in daily practice of the dermatological examination assisting the diagnosis and assessing the degree of invasion and activity of skin diseases.

\section{HISTORY}

Jacques and Pierre Curie, in 1881, developed the principle of ultrasound when they discovered the isoelectric characteristics of certain crystals. ${ }^{13}$ They observed that when a force is applied on the crystal surface, it produces an electric discharge and, if an electric signal is applied on the same surface, the crystal undergoes expansion or contraction, depending on its polarity. Alternating electric fields produce vibration of the crystal causing sound waves of high frequency.

The ultrasound image was based on the principle of sonar used on ships during World War II. At this time also began its medical use, with black and white images. In 1971, Kossoff introduced the grayscale. Thus, different echo intensities reproduce, on the

Study performed at Departamento de Radiologia of the Faculdade de Medicina of the Universidade Federal do Rio de Janeiro (FM-UFRJ) and at Serviço de Dermatologia of the Faculdade de Ciências Médicas da Universidade do Estado do Rio de Janeiro (FCM-UERJ) - Rio de Janeiro (RJ), Brazil. 
screen, different shades of gray. The pioneers in using ultrasound in dermatology were Alexander and Miller, in 1979, who used a unidirectional device (A-mode) to measure skin thickeness. ${ }^{14}$ In the $80 \mathrm{~s}$, emerged the equipment with B-mode, bidirectional, increasing its application in dermatology. ${ }^{13,15}$

The development of new electronics components and the introduction of techniques for digital analysis allowed the improvement of the image, essential for the diagnostic accuracy.

\section{PRINCIPLES}

A basic knowledge of the principle of ultrasound is essential for a correct interpretation of the image.

The main component of an ultrasound device is called transducer. Transducers have a material (usually piezoelectric crystals) that generates acoustic waves when subjected to an electric voltage. ${ }^{16}$ This wave propagates in fluid and/or surrounding tissue and the echoes (back waves) reflected by these structures return to the transducer, which transforms them into electrical energy. Thus, the transducer is the element that transforms electrical energy into mechanical acoustic wave and makes the reverse process when receiving the echoes, being the transmitter and the receiver component. ${ }^{17,18}$

The electrical energy, after being processed by the computer, causes the formation of an image view at the monitor. This is represented on the screen by luminous points that vary in intensity according to the degree of reflection of different structures that the sound beam crosses. This variation is what makes ultrasound an excellent method for assessing edges and interface between different regions. ${ }^{7}$

In two-dimensional-ultrasound (B-mode), the brightness of each pixel corresponds to the amplitude of the echo. ${ }^{18}$ The intensity of the echoes in the image is called echogenicity. Images with high intensity echoes are called echogenic or hyperechoic; those with low intensity are called hypoechoic; and the ones without echoes, anechoic or echolucent. What determines the echogenicity of each tissue is the speed at which the sound wave can pass through it and the quantity and intensity of echoes returned to the device. In normal skin, the echogenicity of each layer depends on its main component that, in the epidermis, is the keratin; in the dermis, is the collagen; and in subcutaneous tissue, is the fat lobules. ${ }^{19}$ The interval between the emitted and reflected waves allows to calculate the distance between the reflected structures.

The images are obtained in vertical sections and both the resolution and penetration vary according to the frequency. ${ }^{16,20}$ The higher the frequency, the shorter the wavelength that reaches the tissues: better reso- lution and less penetration. In dermatology, high resolution devices with high frequency transducers are used. ${ }^{16}$ Devices of 20 to $25 \mathrm{MHz}$ enable the study of the dermis and epidermis and are those with the best resolution for the observation of surface structures. Frequencies between 50 and $100 \mathrm{MHz}$ present little penetration, limited to the epidermis. ${ }^{7}$

Doppler is based on the principle that sound waves emitted by the transducer and by the reflector move in relation to each other. Thus, the Doppler is used to moving structures, such as the blood flow. On color Doppler the blood flow is differentiated by colors while the power Doppler shows the flow volume. ${ }^{7}$ Technological advances increased the sensitivity of color Doppler, eliminating the use of contrast medium and reducing the risk of potential adverse events. ${ }^{21}$

\section{TECHNICAL CONSIDERATIONS}

The skin ultrasound examination starts with thorough inspection of the site to be examined. Subsequently, a thick layer of gel is applied between the skin and the transducer to obtain a best focal point. Thus, the residual vegetative lesions can better defined.

It's important the use of sensitive transducer, which fits the skin contour of different body segments such as face and distal phalanx. The contact of the transducer with the skin should be as smooth as possible to avoid compression of the anatomical structures that, in this tissue, are superficial and thin.

For the examination of the nails, the fingers should be fully extended. A gelatinous cushion can be used to study the nail unit. ${ }^{21}$

The trichotomy of a hairy area to be studied should be made preferably with blade and not with scissors, allowing better contact between the transducer and the skin. The hair shaft can also be assessed in the HFUS.

To study the lesions that have severe crusting or keratinization it is recommended to remove them, since they cause attenuation of the sound beam, reducing the accuracy of the test.

An appropriate dermatologic evaluation using HFUS includes discriminating the exact topography to be studied, differentiating the skin layers, their thickness and vascularization, and possible associated pathological findings. ${ }^{19}$ Lesions, if present, should be evaluated regarding three-dimensional size, thickness and depth, morphology, content (cystic, solid or mixed), foci of calcification or necrosis, vascularization, exact location and involvement of adjacent structures. ${ }^{22}$

\section{ULTRASONOGRAPHY OF THE NORMAL SKIN}

To identify skin changes with HFUS is necessary, first, to understand the ultrasonographic findings of normal skin. 
HFUS with frequency higher than $20 \mathrm{MHz}$ clearly reflects the different layers of the skin. ${ }^{11}$ The epidermis is presented as a hypoechoic line; the dermis, as a hyperechoic band, less shiny than the epidermis; and the subcutaneous tissue, as a hypoechoic layer with the presence of hyperechoic longitudinal structures corresponding to fibrous septa therein (Figure 1) ${ }^{19}$ However, echogenicity and thickness of the dermis are variable depending on the age of the patient. In newborns, it is slightly hypoechoic, as in the elderly or in individuals with intense actinic damage, it is observed, between the epidermis and the dermis, a hypoechoic line called epidermal band of low echogenicity - sonographic representation of elastosis and laxity of the papillary dermis. ${ }^{23,24}$

Skin layers of the scalp are similar to other sites. However, hypoechoic structures arranged obliquely are observed, corresponding to hair follicles. Depending on the stage of the hair cycle, these structures may be observed in the dermis or subcutaneous tissue (Figure 2). ${ }^{25}$
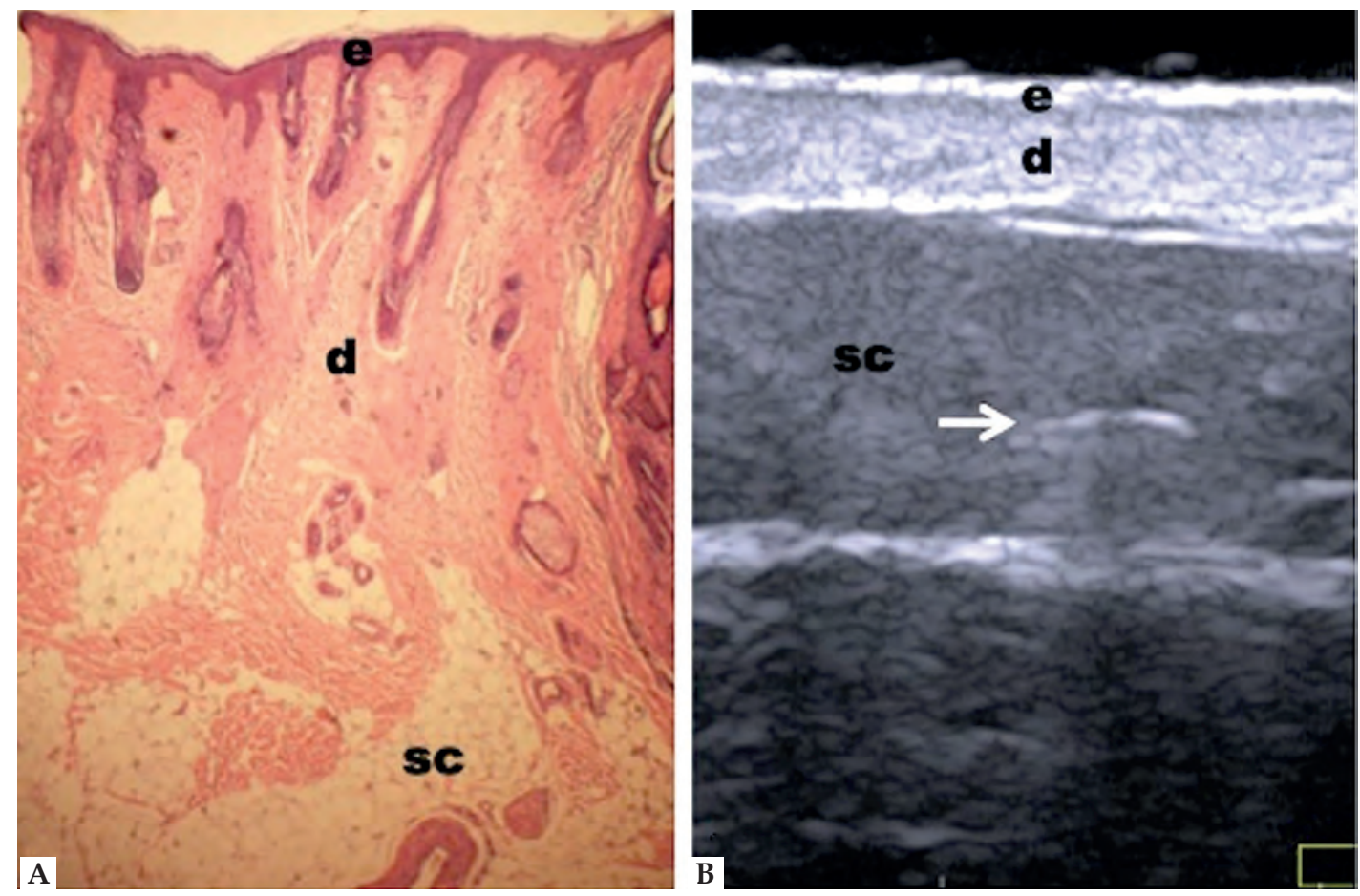

Figure 1:

Non-glabrous

skin anatomy.

(A) Normal

skin histology.

(B) HFUS,

transverse view.

(e) Epidermis.

(d) Dermis. (sc)

Subcutaneous

tissue with the presence of fibrous septa $(\Rightarrow)$

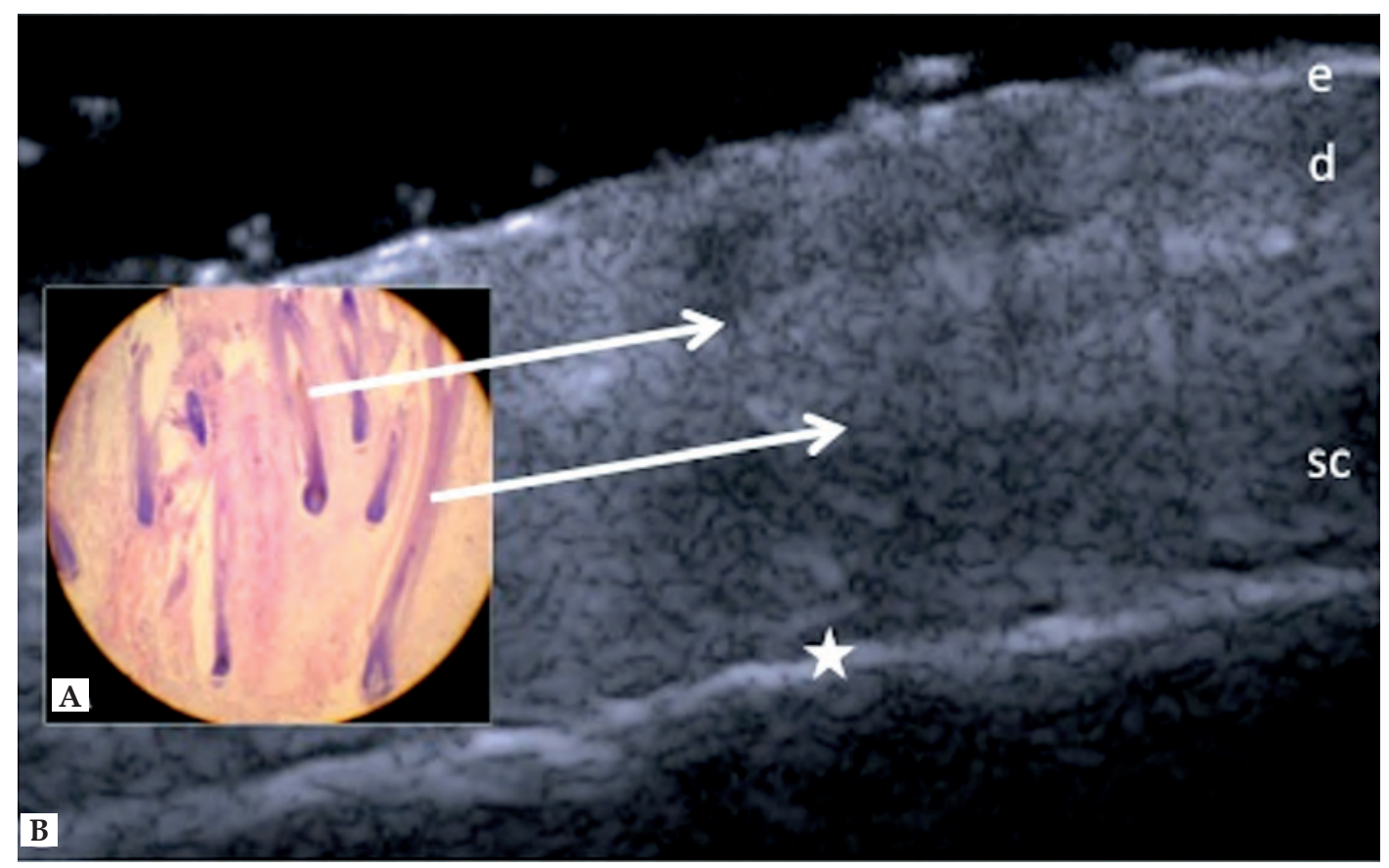

Figure 2:

Scalp. (A)

Histology,

longitudinal

section. (B) HFUS,

longitudinal view.

(e) Epidermis.

(d) Dermis. (sc)

Subcutaneous

tissue. $(\Rightarrow)$

Hypoechoic

oblique bands

corresponding to

hair follicles. $(\star)$

Margin of cranial

bone 
In palmoplantar region, the epidermis appears as hypoechoic bilaminar structure, which may be the result of the contrast between the skin itself and the very thick and compact stratum corneum. ${ }^{17,19}$

Nail unit structures are well differentiated in HFUS. The nail plate presents bilaminar aspect due to ventral and dorsal plates. Below the nail plate, there is a hypoechoic area that corresponds to the nail bed. In the proximal part of this region is the nail matrix, which is echogenic. Below the nail bed, there is a strongly hyperechoic line corresponding to the distal phalanx bone (Figure 3). ${ }^{19,26}$

\section{APPLICATIONS}

\section{SKIN TUMORS}

In HFUS, skin cancer, malignant or benign, usually presents as hypoechoic areas in contrast to the adjacent healthy tissue. ${ }^{18,27}$ In addition to echogenicity, it's possible to assess the lesion shape, the longitudinal, transverse and axial measures, the edges, the contour and the involvement of deep layers, such as muscle, cartilage and bone. ${ }^{28}$ The study of the vascularization can be accomplished with the combination of color Doppler or power Doppler, which allows ob-

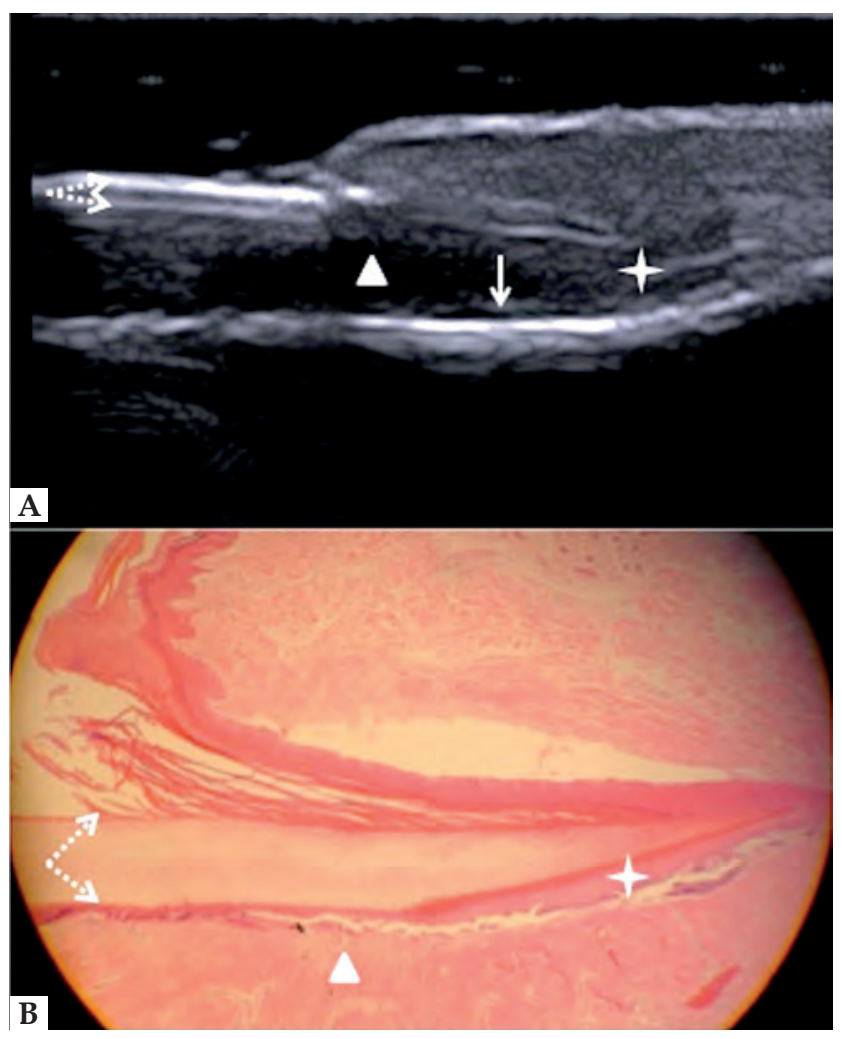

FIGURE 3: Normal nail unit, longitudinal section. (A) HFUS. (B) Histological section. ( $\rightarrow$ )Floor plate.

( $)$ Dorsal plate. $(\star)$ Nail matrix. $(\Delta)$ Nail bed and $(\boldsymbol{\nabla})$ distal phalanx bone serving the distribution, size and nature (arterial or venous) of tumor vessels. ${ }^{29}$

As the cutaneous lesions may be asymmetric, the measurement of the tumor thickness should be based on the site of greater invasion.

Some skin tumors can be distinguished by ultrasound image. Basal cell carcinoma (BCC) presents as a hypoechoic area, well defined, with irregular contour, usually located in the dermis, but may extend to deep planes. ${ }^{30}$ Often the presence of hyperechoic spots inside the tumor is observed. These images are attributed to the presence of corneal cysts, microcalcifications and clusters of apoptotic cells within the tumor mass (Figure 4). ${ }^{18,31}$ There are reports in the literature of subclinical satellite lesions that were diagnosed using HFUS. ${ }^{30}$ The intra and peritumoral blood flow is discrete and consists of arteries and veins of low flow. ${ }^{29}$ To evaluate the morphology, vascularization and thickness of BCC, HFUS can be an excellent tool in choosing the therapeutic approach. It provides a detailed preoperative study, reducing the number of incompletely excised lesions and avoiding large resection, which could lead to functional and aesthetic problems. In non-surgical treatments, such as photodynamic therapy and immunomodulators, it allows the monitoring of the treatment in a noninvasively manner.

Although the appearance of BCC and squamous cell carcinoma (SCC) are similar in HFUS, it is possible to suggest the distinction between these two tumors, since the latter seldom presents hyperechoic points within the tumor. SCC, due to its more aggressive behavior, is more likely to invade soft tissues, cartilage and adjacent bone. In color Doppler mapping, there is a mixed pattern with internal and peripheral vascularization. Marmur et al point out that, due to the SCC characteristic of generally presenting hyperkeratosis and to the higher inflammatory process associated, the tumor area can be overestimated when evaluated by ultrasound. ${ }^{18}$

In melanoma, HFUS is used to establish the tumor thickness, margins and vascularization. ${ }^{32}$ In the ultrasound image, melanoma usually presents itself as a homogeneous hypoechoic area, oval or spindle-shaped (Figure 5). Despite nevic lesions present irregular echogenicity and melanoma, homogeneous, these lesions cannot be differentiated by HFUS, which may overestimate tumor size in lesions with nevus-melanoma association. ${ }^{33,34}$ In ulcerated lesions, skin may be irregular. ${ }^{35}$ The mapping of melanocytic lesions with color Doppler shows that, in melanoma, vascularization is more intense than in benign lesions, predominating arterial vessels of low flow. Bessoud et al, in a study of pigmented skin lesions, showed that the detection of intralesional blood vessels has $100 \%$ spec- 
ificity and 34\% sensitivity in distinguishing between melanoma and non-melanoma tumors. ${ }^{36}$ The metastatic potential of these lesions can also be estimated by the study of tumor angiogenesis. ${ }^{35,36,37}$ Samimi et al demonstrated that HFUS can be used to differentiate
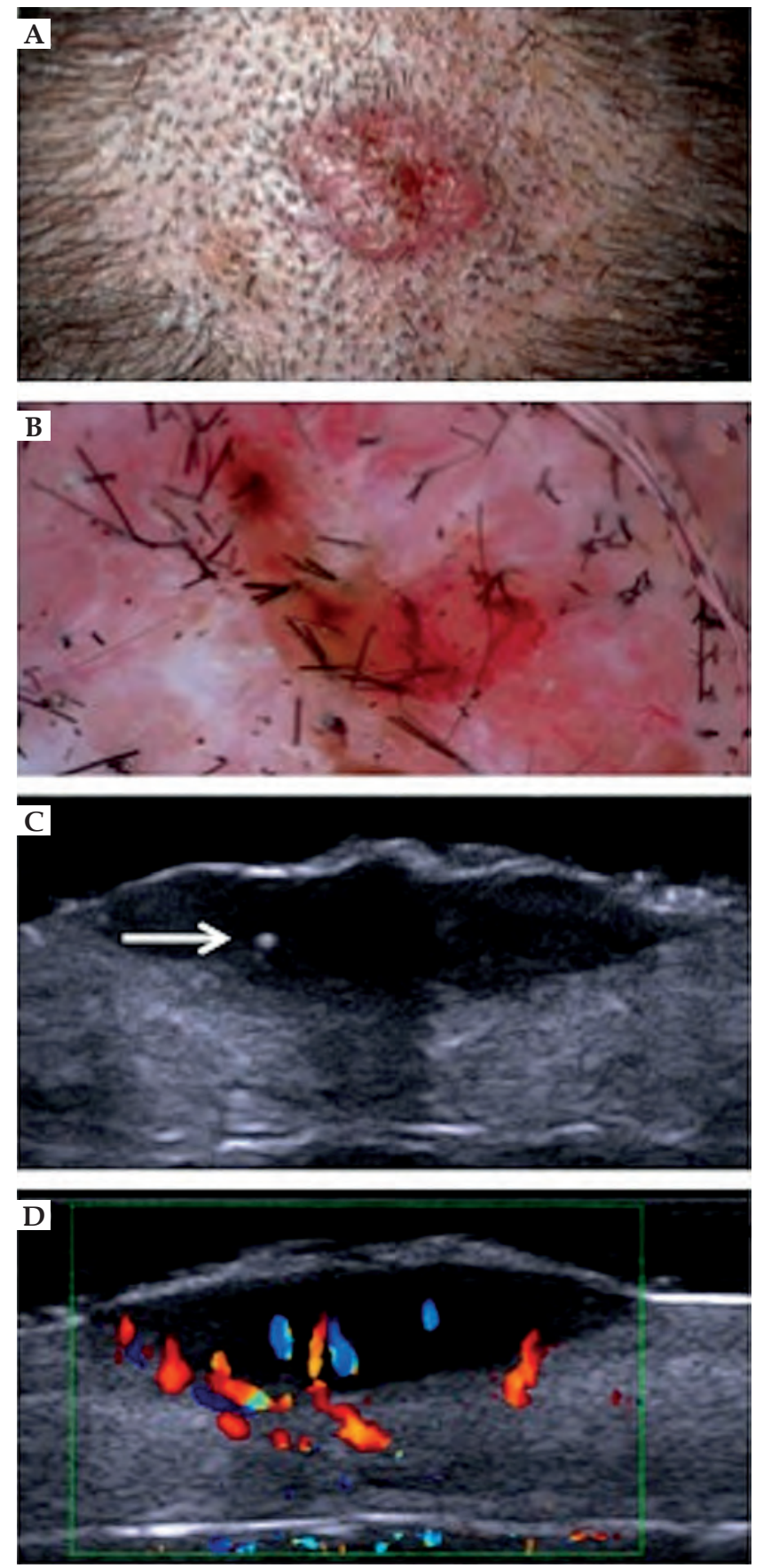

Figure 4: Basal cell carcinoma in the scalp. (A) Clinical aspect. (B) Dermatoscopy presenting arboriform vessels. (C) HFUS, transversal view. Hyperechoic point within the lesion $(\Rightarrow)$. (D) Color Doppler. Blood vessels inside and at the base of the lesion (blue and red)

metastatic melanoma from blue nevus in patients with a history of melanoma presenting homogeneous blue lesions on dermoscopy, eliminating unnecessary excisions nevus. In the ultrasound image, the nevic lesions present discoid shape and are located in the dermis, while the metastatic lesions have irregular shape and are situated in the hypodermis (Figure 6). ${ }^{28}$ Solivetti et al suggest that, to detect metastatic lesions, a transducer of different frequency is necessary because, depending on the site and size of a lesion, it can be well observed in a particular frequency and be completely invisible in another. ${ }^{38}$

Ulrich et al showed that assessment with HFUS to detect melanoma metastases in regional lymph nodes has better sensitivity and specificity than clinical examination, which can be very relevant due to the 


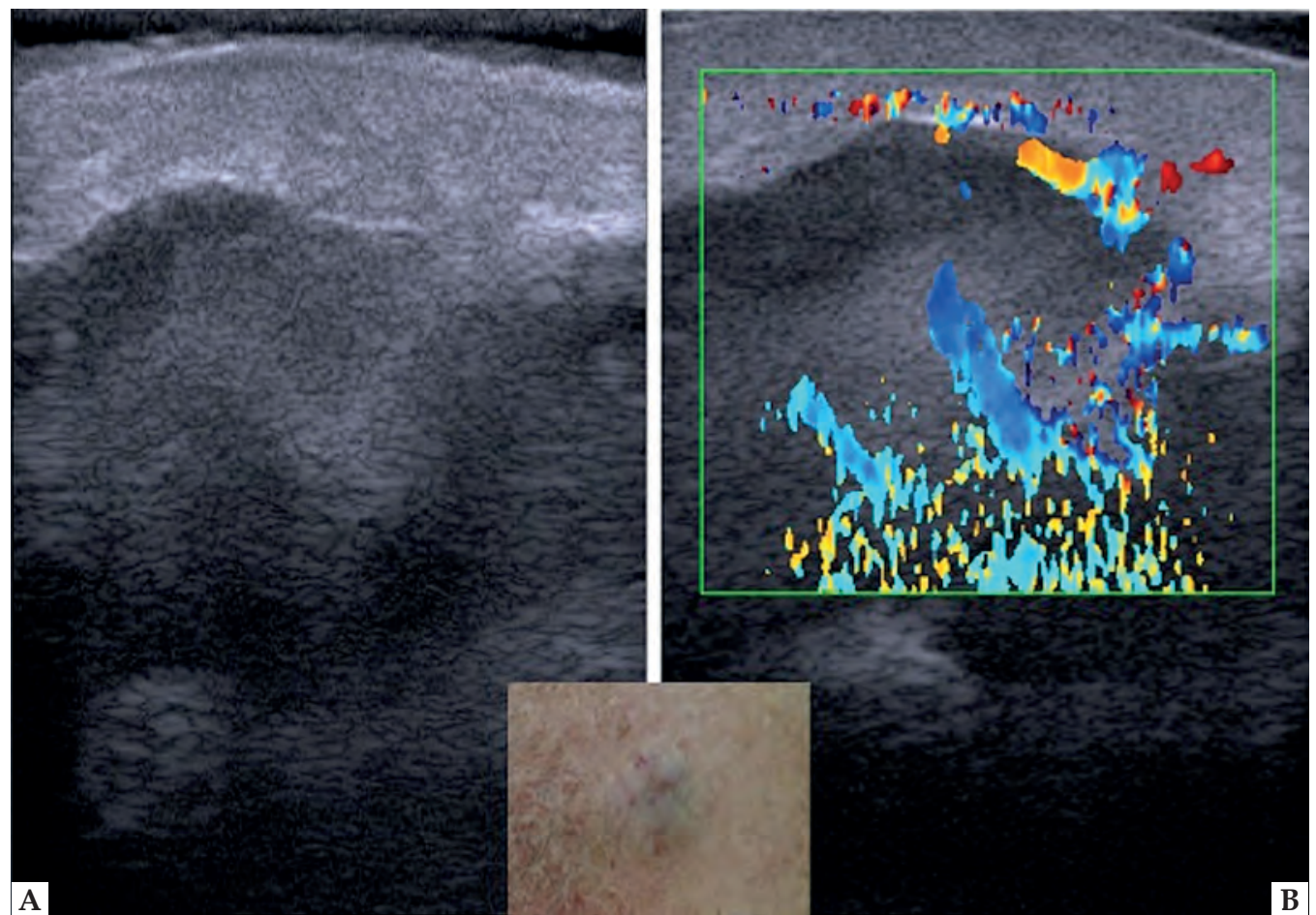

Figure 6: Metastatic melanoma. HFUS, longitudinal view. Epidermis and dermis with normal appearance. In the subcutaneous tissue, irregular lesion with variable echogenicity. (B) Color Doppler. Intralesional vascularity recent introduction of new drugs for the treatment of metastatic melanoma. ${ }^{39,40}$ Metastatic lymph nodes, in the ultrasound, are round, with clear edges and hypoechoic or echolucent center (necrosis), whereas reactive lymphadenopathy present elliptical aspect with hyperechoic center and central vascularization., 38,41

Some factors may lead to error in measurement of tumor thickness with HFUS. Inflammatory process associated with cancer or prior to the examination procedures, presence of hypertrophic perilesional glands and nevus-melanoma association may overestimate its size while the presence of ulceration may underestimate it. $29,31,42,43$

\section{INFLAMMATORY AND INFECTIOUS DISEASES}

In systemic sclerosis (SS), the differentiation between the stages of the disease (edematous, fibrotic and atrophic) is critical for treatment planning, and skin involvement can estimate the participation of internal organs and the patient's prognosis. ${ }^{44,45,46}$ Thus, for an overall evaluation of the SS a thorough study of skin changes is critical. ${ }^{47}$ To measure the extent of hardening of the skin, the modified Rodnan score is used, but an objective and reproducible method, which differentiate from edema, is critical to managing the disease. HFUS enables monitoring the developments of SS and the proposed treatment in a serial and non-invasive manner. Low echogenicity and increased skin thickness represent the edema in an initial phase while the decrease of skin thickness and increased echogenicity correspond to fibrosis and thinning..$^{45}$ Color Doppler allows the study of the digital arteries dynamically, being an excellent method to evaluate the peripheral vascular disease. ${ }^{47}$

Just like in SS, controlling the activity and extent of disease in morphea is important in the selection of treatment. In HFUS, echogenicity follows the activity of the framework: inflammatory lesions are hypoechoic while sclerotic are hyperechoic. ${ }^{48} \mathrm{Li}$ et al highlighted the importance of this imaging method in the follow-up of children with morphea, because the skin texture changes throughout its development, hindering clinical monitoring. ${ }^{99} \mathrm{~A}$ wide scan of the affected area with HFUS enables the detection of subclinical lesions allowing early treatment. This method can also be used to assess the effectiveness of the treatment. ${ }^{50}$ On color Doppler mapping, detection of increased skin blood flow can be considered a highly sensitive and specific finding in disease activity. ${ }^{51}$

In the evaluation of psoriasis, the HFUS associated with color Doppler imaging can be used to assess disease activity and response to treatment. In psoriatic plaque, the most common ultrasound finding is thickening of the epidermis and dermis due to the increased proliferation of keratinocytes and accumulation of inflammatory cells. ${ }^{52}$ As skin thickness varies by anatomical site, comparative study with healthy contralateral area is necessary (Figure 7). During the 
active phase, in HFUS, it's possible to observe a subepidermal hypoechoic line and, in color Doppler, there is an increased blood flow in the dermis. Gutierrez et al proposed that the study of the microcirculation can be a marker of activity of the psoriasis..$^{53}$ The ultrasonographic changes of the nail apparatus are loss of the bimorph aspect of the nail plate and presence of pittings or irregularities on the nail surface. ${ }^{54}$
Plantar warts can be easily detected by HFUS. The interruption of the epidermis can be observed, as well as the presence of hypoechoic lesions affecting the epidermis and dermis, and in most cases, increased arterial blood flow in the dermis (Figure 8). Thus, HFUS provides accurate information on the location and extension, enabling appropriate therapeutic choice. ${ }^{55}$

Clinical manifestations of active and scarring
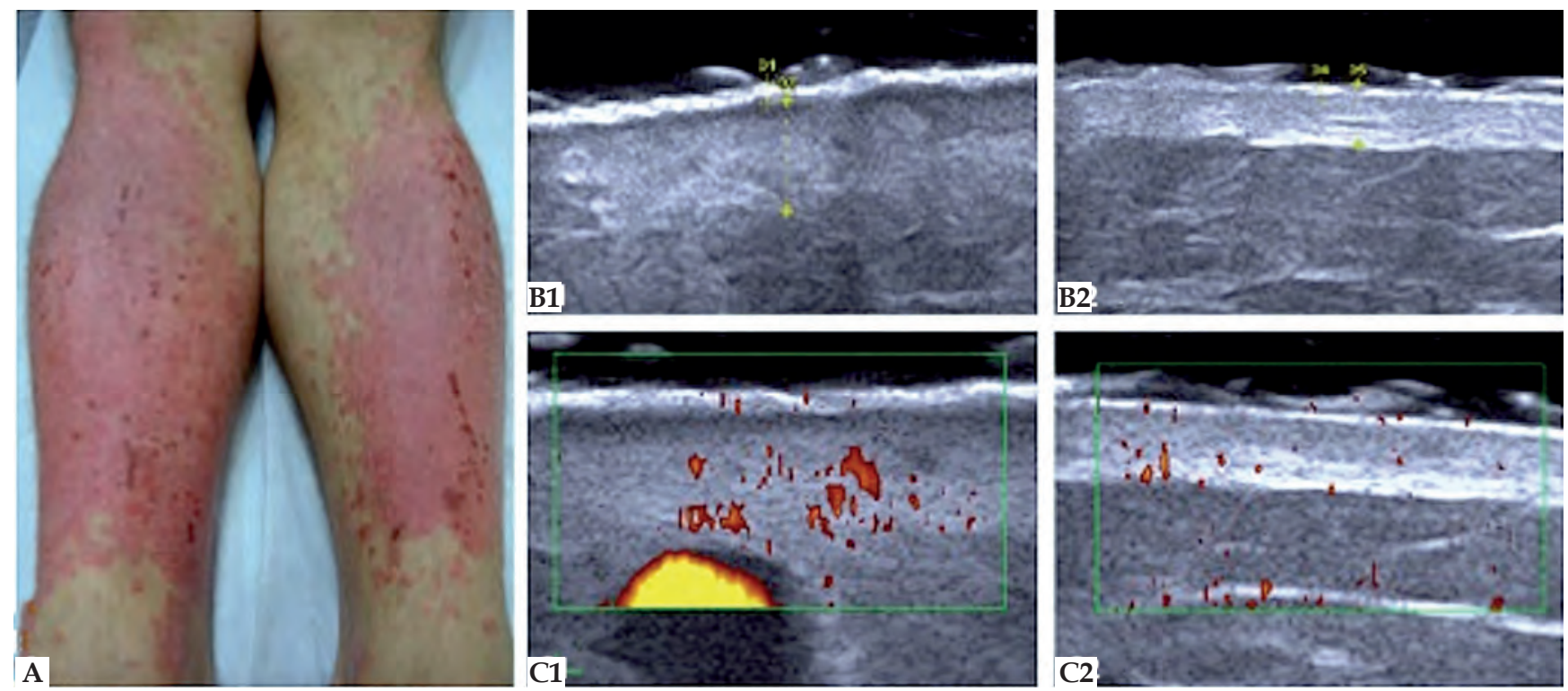

Figure 7: Psoriasis. (A) Posterior region of the legs. (B) HFUS, transversal view. (B1) Psoriasis plaque. Epidermis with $0.50 \mathrm{~mm}$ thickness and dermis with $2.56 \mathrm{~mm}$ thickness. (B2) Healthy contralateral region. Epidermis with $0.34 \mathrm{~mm}$ thickness and dermis with $1.67 \mathrm{~mm}$ thickness. (C) Color Doppler. (C1) Increased blood flow of the affected area, setting up disease activity. (C2) Healthy contralateral region
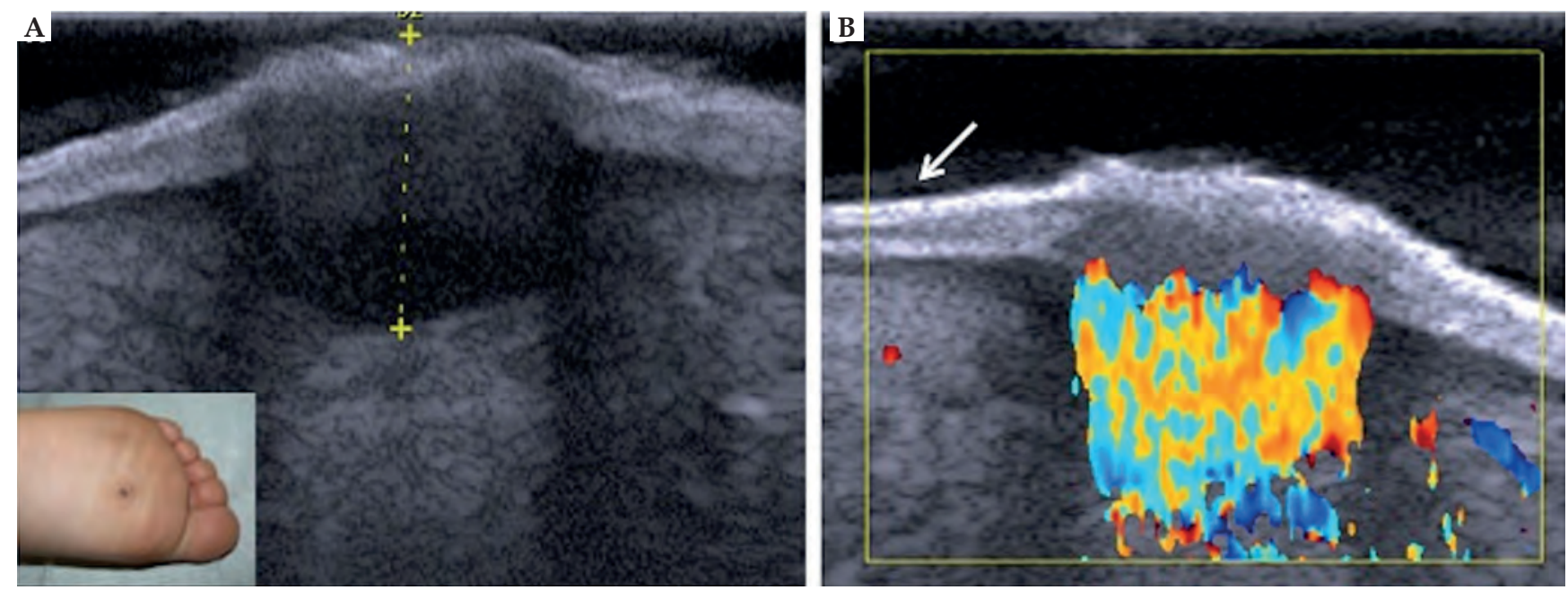

FigURE 8: Plantar wart. (A) HFUS, transversal view. Fusiform hypoechoic lesions localized in the epidermis and in the dermis. (B) Color Doppler. Intense vascularization within the lesion with predominance of arterial vessels. ( $\llcorner$ ) Ultrasonographic bilaminar aspect of the plantar region skin 
acne can be studied in HFUS. The degree of inflammatory reaction can be monitored with color Doppler and ultrasound study provides the size, depth and morphology of scar lesions. ${ }^{56}$

Wortsman et al assessed areas affected by hidradenitis and observed increased thickness and decreased echogenicity of the skin. With the HFUS, it is also possible to detect subclinical lesions and fistulas. Color Doppler shows increased vascularity. ${ }^{57}$

\section{AGING SKIN AND COSMIATRY}

HFUS can be used to assess, quantify and classify skin aging. ${ }^{24}$ Intrinsic aging (senility) has as main histological features thinning of the dermis with collagen decrease, elastic fibers degeneration and less hydration. In HFUS, these changes are consistent with alterations in echogenicity of the dermis, which is directly related to the density of the extracellular matrix. In photoaging, the most relevant histopathological findings are solar elastosis, collagen architecture change and glycosaminoglycan accumulation. These changes are reflected in the ultrasound image, in the formation of a subepidermal low echogenicity band (SLEB) (Figure 9) ${ }^{58,59}$ However, as the echogenicity of the dermis and SLEB are subject to individual variations, as hydration and anatomical site, new studies with high frequency and good resolution equipment are needed so that these parameters can be used as aging markers. ${ }^{60}$

In dermal filling, HFUS allows an effective control of the location of the applied product and volume of skin deposits, which can improve the quality of the procedure. ${ }^{61,62}$ Kohn et al, evaluating procedures with hyaluronic acid, concluded that ultrasound morphology depends on the injection plan. In the dermis, there is an increase in echogenicity as subdermal injections produce multiple anechoic pearls. ${ }^{63}$ De Pasquale et al observed that, in the subcutaneous tissue, hyaluronic acid is perceived as a regular, well defined, hypoechoic mass. ${ }^{64}$ As different fillers have their own characteristics to HFUS, this may also be useful to differentiate their nature so as to detect possible complications. ${ }^{61}$

\section{OTHERS}

Grap et al, using HFUS to early detect pressure ulcers, concluded that changes in thickness and echogenicity of the skin layers are markers of tissue damage and precede the onset of clinically visible lesions. ${ }^{65}$

In skin diseases that cause skin thinning, as linear atrophoderma of Moulin, HFUS can be useful both in the evaluation and in the follow up of these patients because it enables the comparative study of contralateral anatomical region, which is not affected. ${ }^{66}$

A study by Goldberg et al, with patients with

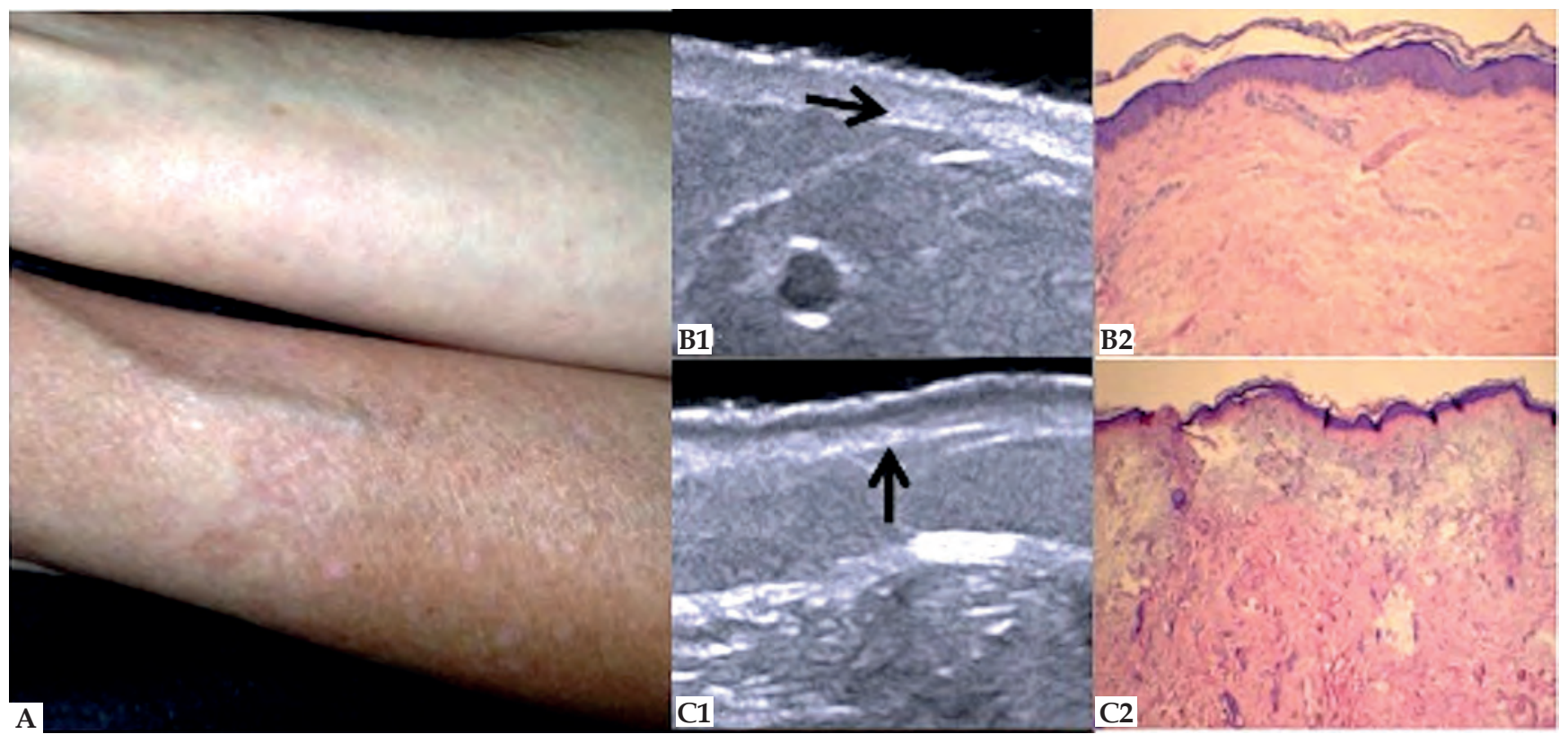

Figure 9: Photoaging. (A) Ventral and dorsal region of the forearm. (B1) HFUS, longitudinal view. Decreased dermis echogenicity $(\rightarrow)$. (B2) Discrete degeneration of collagen fibers. Hematoxylin \& Eosin 10X. (C1) HFUS, longitudinal view. Subepidermal low echogenicity band (A). (C2) Solar elastosis. Hematoxylin \& Eosin 10X 
plantar hyperkeratosis, showed that congenital pachyonychia (CP), due to the formation of blisters, can be differentiated, in HFUS, from the Meleda disease and from epidermolytic palmoplantar keratoderma. In CP is observed, between the epidermis and the dermis, an anechoic layer corresponding to the blister liquid, which supports the diagnosis and justifies the pain reported by patients. ${ }^{67}$

\section{CONCLUSION}

At a time when different techniques of diagnostic imaging converge to the improvement of skin analysis, HFUS provides useful information for the diagnosis, monitoring and treatment of physiological and pathological changes of the skin and its appendages.

However, because its application is relatively new in dermatology, further studies are needed so that terminology and evolutionary analysis criteria can be standardized, expanding its practice in dermatology routine. $\square$

\section{REFERENCES}

1. Mogensen M, Morsy HA, Thrane L, Jemec GB. Morphology and epidermal thickness of normal skin imaged by optical coherence tomography. Dermatology. 2008;217:14-20.

2. Lallas A, Giacomel J, Argenziano G, García-García B, González-Fernández D, Zalaudek I, et al. Dermoscopy in general dermatology:practical tips for the clinician. Br J Dermatol. 2014;170:514-26.

3. Giacomel J, Lallas A, Argenziano G, Reggiani C, Piana S, Apalla Z, et al. Dermoscopy of basosquamous carcinoma. Br J Dermatol. 2013;169:358-64.

4. Longo C, Farnetani F, Ciardo S, Cesinaro AM, Moscarella E, Ponti G, et al. Is confocal microscopy a valuable tool in diagnosing nodular lesions? A study of 140 cases. Br J Dermatol. 2013;169:58-67.

5. Soyer HP, Prow TW. Reflectance confocal microscopy in the diagnosis of nodular skin lesions. Br J Dermatol. 2013;169:4.

6. Sattler E, Kästle R, Welzel J. Optical coherence tomography in dermatology. J Biomed Opt. 2013;18:061224.

7. Kleinerman R, Whang TB, Bard RL, Marmur ES. Ultrasound in dermatology. Principles and applications. J Am Acad Dermatol. 2012;67:478-87.

8. Barcaui EO, Carvalho ACP, Piñeiro-Maceira J, Valiante PM, Barcaui CB. Ultrassonografia de alta frequência $(22 \mathrm{MHz})$ na avaliação de neoplasias cutâneas malignas. Surg Cosmet Dermatol. 2014;6:105-11.

9. Ulrich J, Schwürzer-Voit M, Jenderka KV, Voit C. Sonographic diagnostics in dermatology. J Dtsch Dermatol Ges. 2014;12:1083-98

10. Lucas VS, Burk RS, Creehan S, Grap MJ. Utility of High-frequency ultrasound: moving beyond the surface to detect changes in skin integrity. Plast Surg Nurs. 2014;34:34-8.

11. Wortsman $X$, Wortsman J. Clinical usefulness of variable-frequency ultrasound in localized lesions of the skin. J Am Acad Dermatol. 2010;62:247-56.

12. Wortsman X. Common application of dermatologic sonography. J Ultrasound Med. 2012;31:97-111

13. Nemade $\mathrm{H}$, Rao A, Thompson P. From bats and ships to prostate and kidneys. A brief history of ultrasound in urology. J Urol. 2013;189:452.

14. Alexander $\mathrm{H}$, Miller DL. Determining skin thickness with pulsed ultrasound. $J$ Invest Dermatol. 1979;72:17-9.

15. Resende CMC. Artefatos em ultrassonografia e suas bases físicas. Rio de Janeiro: Revinter; 1988.

16. Bagatin E, Caetano LVN, Soares JLM. Ultrasound and dermatology: basis principles and main applications in dermatologic research. Expert Rev of Dermatol. Oct. 2013;8:463-77.

17. Wortsman X. Ultrasound in dermatology: why, how, and when? Semin Ultrasound CT MR. 2013;34:177-95.

18. Marmur ES, Berkowitz EZ, Fuchs BS, Singer GK, Yoo JY. Use of high frequency, high resolution ultrasound before Mohs surgery. Dermatol Surg. 2010;36:841-7.

19. Barcaui EO, Carvalho ACP, Piñeiro-Maceira J, Barcaui CB, Moraes H. Estudo da anatomia cutânea com ultrassom de alta frequência $(22 \mathrm{MHz})$ e sua correlação histológica. Radiol Bras. 2015;48:324-9.

20. Ulrich M, Stockfleth E, Roewert-Huber J, Astner S. Noninvasive diagnostic tools for nonmelanoma skin cancer. Br J Dermatol. 2007;157:56-8.

21. Wortsman $X$. Sonography of cutaneous and ungual lumps and bumps. Ultrasound Clin. 2012;7:505-523.
22. Gaitini D. Introduction to color Doppler ultrasound of the skin. In: Wortsmax X, Jemec GBE. Dermatologic ultrasound with clinical and histologic correlations. New York: Springer; 2013. p.3-14.

23. Gniadecka M. Effects of aging on dermal echogenicity. Skin Res Technol. 2001;7:204-7.

24. Crisan D, Lupsor M, Boca A, Crisan M, Badea R. Ultrasonographic assessment $f$ skin structure according to age. Indian J Dermatol Venereol Leprol. 2012;78:519.

25. Wortsman X, Wortsman J, Matsuoka L, Saavedra T, Mardones F, Saavedra D, et al. Sonography in pathologies of scalp and hair. Br J Radiol. 2012;85:647-55

26. Wortsman X, Jemec GB. Ultrasound Imaging of nails. Dermatol Clin. 2006;24:323-8.

27. Barcaui Ede 0, Carvalho AC, Valiante PM, Barcaui CB. High-frequency ultrasound associated with dermoscopy in pre-operative evaluation of basal cell carcinoma. An Bras Dermatol. 2014;89:828-31.

28. Samimi M, Perrinaud A, Naouri M, Maruani A, Perrodeau E, Vaillant $L$, et al High-resolution ultrasonography assists the differential diagnosis of blue naevi and cutaneous metastases of melanoma. Br J Dermatol. 2010;163:550-6.

29. Wortsman X. Sonography of facial cutaneous basal cell carcinoma. A first-line imaging technique. J Ultrasound Med. 2013;32:567-72.

30. Bobadilla F, Wortsman X, Muñoz C, Segovia L, Espinoza M, Jemec GB. Présurgical high resolution of facial basal cell carcinoma: Correlation with histology. Cancer Imaging. 2008:8:163-72.

31. Desai TD, Desai AD, Horowitz DC, Kartono F, Wahl T. The use of high-frequency ultrasound in the evaluation of superficial and nodular basal cell carcinoma. Dermatol Surg. 2007;33:1220-7

32. Guitera P, Menzies SW. State of the art of diagnostic technologyfor early-stage melanoma. Expert Rev Anticancer Ther. 2011;11:715-23.

33. Psaty EL, Halpern AC. Current and emerging technologies in melanoma diagnosis: the state of the art. Clin Dermatol. 2009;27:35-45.

34. Kaikaris V, Samsanavičius D, Kęstutis Maslauskas, Rimdeika R, Valiukevičienè $\mathrm{S}$, Makštienė J, et al. Measurement of melanoma thickness - comparison of two methods: ultrasound versus morphology. J Plast Reconstr Aesthet Surg. 2011;64:796-802

35. Wortsman X. Sonography of the primary cutaneous melanoma: a review. Radio Res Pract. 2012;2012:814396.

36. Bessoud B, Lassau N, Koscielny S, Longvert C, Avril MF, Duvillard P, et al. Highfrequency sonography and color doppler in the management of pigmented skin lesions. Ultrasound Med Biol. 2003;29:875-9.

37. Machet L, Belot V, Naouri M, Boka M, Mourtada Y, Giraudeau B, et al. Preoperative measurement of thickness of cutaneous melanoma using high-resolution $20 \mathrm{MHz}$ ultrasound imaging: a monocenter prospective study and systematic review of the literature. Ultrasound Med Biol. 2009:35:1411-20.

38. Solivetti FM, Di Luca Sidozzi A, Pirozzi G, Coscarella G, Brigida R, Eibenshutz L. Sonographic evaluation of clinically occult in-transit and satellite metastases from cutaneous malignant melanoma. Radiol Med. 2006;111:702-8.

39. Krüger U, Kretschmer L, Thoms KM, Padeken M, Peter Bertsch H, Schön MP, et al. Lymph node ultrasound during melanoma follow up significantly improves metastasis detection compared with clinical examination alone: a study on 433 patients. Melanoma Res. 2011;21:457-63.

40. Gedye C, Hogg D, Butler M, Joshua AM. New treatments for metastatic melanoma. 
CMAJ. 2014;186:754-60.

41. Machet L, Nemeth-Normand F, Giraudeau B, Perrinaud A, Tiguemounine J, Ayoub $J$, et al. Is ultrasound lymph node examination superior to clinical examination in melanoma follow up? A monocentre cohort study of 373 patients. Br J Dermatol. 2005; $152: 66-70$

42. Nassiri-Kashani M, Sadr B, Fanian F, Kamyab K, Noormohammadpour P, Shahshahani MM, et al. Pré-operative assessment of basal cell carcinoma dimensions using high frequency ultrasonography and its correlation with histopathology. Skin Res Technol. 2013;19:e132-8.

43. Crisan M, Crisan D, Sannino G, Lupsor M, Badea R, Amzica F. Ultrasonographic staging of cutaneous malignant tumors: an ultrasonographic depth index. Arch Dermatol Res. 2013;305:305-13.

44. Porta F, Gargani L, Kaloudi O, Schmidt WA, Picano E, Damjanov N, et al. The new frontiers of ultrasound in the complex world of vasculitides and scleroderma. Rheumatology (Oxford). 2012;51:vii26-30.

45. Hesselstrand R, Scheja A, Wildt M, Akesson A. High-frequency ultrasound of skin involvement in systemic sclerosis reflects oedema, extension and severity in early disease. Rheumatology (Oxford). 2008;47:84-7.

46. Hughes M, Herrick A. Systemic sclerosis. Br J Hosp Med (Lond). 2012;73:50910, 511-6

47. Gutierrez M, Pineda C, Cazenave T, Piras M, Erre GL, Draghessi A, et al. Ultrasound in systemic sclerosis. A multi-target approach from join to lung. Clin Rheumatol. 2014;33:1039-47.

48. Nouri S, Jacobe $\mathrm{H}$. Recent developments in diagnosis and assessment of morphea. Curr Rheumatol Rep. 2013;15:308.

49. Li SC, Liebling MS, Haines KA. Ulrasonography is a sensitive tool for monitoring localized scleroderma. Rheumatology (Oxford). 2007;46:1316-9.

50. Buense R, Duarte IA, Bouer M. Localized scleroderma: assessment of the therapeutic response to phototherapy. An Bras Dermatol. 2012;87:63-9.

51. Wortsman X, Wortsman J, Sazunic I, Carreño L. Activity assessment in morphea using color Doppler ultrasound. J Am Acad Dermatol. 2011;65:942-8.

52. Gutierrez M, Wortsman X, Filippucci E, De Angelis R, Filosa G, Grassi W. High-frequency sonography in the evaluation of psoriasis. J Ultrasound Med. 2009;28:1569-74.

53. Gutierrez M, De Angelis R, Bernardini ML, Filippucci E, Goteri G, Brandozzi G, et al. Clinical, power Doppler sonography and histological assessment of the psoriatic plaque: short-term monitoring in patients treated with etanercept. $\mathrm{Br} \mathrm{J}$ Dermatol. 2011;164:33-7.

54. Aydin SZ, Castillo-Gallego C, Ash ZR, Abignano G, Marzo-Ortega H, Wittmann $\mathrm{M}$, et al. Potential use of optical coherence tomography and high-frequency ultrasound for the assessment of nail disease in psoriasis and psoriatic arthritis. Dermatology. 2013;227:45-51.

55. Wortsman X, Jemec GB, Sazunic I. Anatomical detection of inflammatory changes associated with plantar warts by ultrasound. Dermatology. 2010;220:213-7.

56. Micali G, Tedeschi A, Lacarrubba F, Francesconi L. Clinical marfology and ultrasound correlation in the assessment of acne scars. J Am Acad Dermatol. 2010;62:AB17

57. Wortsman X, Jemec GBE. High frequency ultrasound for the assessment of hidradenitis suppurativa. Dermatol Surg. 2007;33-1-3.

58. Sandby-Møller J, Thieden E, Philipsen PA, Schmidt G, Wulf HC. Dermal echogenicity: a biological indicator of individual cumulative UVR exposure? Arch Dermatol Res. 2004;295:498-504.

59. Sandby-MØller J, Wulf HC. Ultrasonographic subepidermal low-echogenic band, dependence of age and body site. Skin Res Technol. 2004;10:57-63.

60. Gniadecka M, Gniadecki R, Serup J, Søndergaard J. Ultrasound structure digital image analysis of the subepidermal low echogenic band in aged human skin: diurnal changes and interindividual variability. J Invest Dermatol. 1994;102:362-5.

61. Wortsman X, Wortsman J, Orlandi C, Cardenas G, Sazunic I, Jemec GB. Ultrasound detection and identification of cosmetic fillers in the skin. J Eur Acad Dermatol Venereol. 2012;26:292-301.

62. Young SR, Bolton PA, Downie J. Use of high-frequency ultrasound in the assessment of injectable dermal fillers. Skin Res Technol. 2008;14:320-3.
63. Kohn JC, Goh AS, Lin JL, Goldberg RA. Dynamic high-resolution ultrasound in vivo imaging of hyaluronic acid filler injection. Dermatol Surg. 2013;39:1630-6.

64. De Pasquale A, Russa G, Pulvirenti M, Di Rosa L. Hyaluronic acid filler injections for tear-trough deformity: injection technique and high-frequency ultrasound follow-up evaluation. Aesthetic Plast Surg. 2013;37:587-91.

65. Grap MJ, Burk RS, Lucas V, Munro CL, Wetzel PA, Schubert CM. Use of high frequency ultrasound to detect changes in skin integrity: an image evaluation validation procedure. Intensive Crit Care Nurs. 2015;31:141-7.

66. Norisugi 0, Makino T, Hara H, Matsui K, Furuichi M, Shimizu T. Evaluation of skin atrophy associated with linear atrophoderma of Moulin by ultrasound imaging. $J$ Am Acad Dermatol. 2011;65:232-3.

67. Goldberg I, Sprecher E, Schwartz ME, Gaitini D. Comparative study of highresoution multifrequency ultrasound of the plantar skin in patients with various types of hereditary palmoplantar keratoderma. Dermatology. 2013;226:365-70.

\author{
MAILING ADDRESS: \\ Elisa de Oliveira Barcaui \\ Hospital Universitário Clementino Fraga Filho \\ R. Rodolpho Paulo Rocco, 255 \\ Cidade Universitária \\ 21941-913 - Ilha do Fundão - RJ \\ Brazil \\ Email:ebarcaui@gmail.com
}

How to cite this article: Barcaui EO, Carvalho ACP, Lopes FPPL, Piñeiro-Maceira J, Barcaui CB. High frequency ultrasound with color Doppler in dermatology. An Bras Dermatol. 2016;91(3):262-73. 


\section{Questions}

1 - Among the additional tests listed below, choose the option in which the tests enabling the study of skin lesions in real time, non-invasively and without radiation are mentioned:

a) CT, MRI and radiography.

b) CT, optical coherence tomography and ultrasonography.

c) PET-Scan, confocal microscopy and dermoscopy.

d) Confocal microscopy, dermatoscopy and HFUS.

2 - Regarding HFUS equipment, it is incorrect to say that:

a) They have frequency equal to or greater than $15 \mathrm{MHz}$.

b) They are excellent for visualization of abdominal cavity.

c) They have little penetration and excellent resolution for viewing surface structures.

d) With the use of color Doppler, we can estimate the blood flow of skin tissues.

\section{3 - Check the right alternative:}

a) The higher the frequency of the ultrasound transducer, the smaller the wavelength that reaches the tissues.

b) In two-dimensional ultrasound (B-mode), the brightness of each pixel corresponds to echo frequency.

c) The echogenicity of each tissue is inversely proportional to the speed at which the sound wave can pass through it.

d) In normal skin, the echogenicity of each layer is not dependent on its structural member.

4 - When we conduct a skin ultrasound examination, we should consider the following statements, except:

a) The gel layer between the skin and the transducer must be thick.

b) The contact of the transducer with the skin should always be performed by applying pressure to obtain an image with good resolution.

c) In examining one hairy region, shaving must be done preferably with blade.

d) For the examination of the nails, the fingers should stay extended.

5 - Regarding the skin examination with HFUS, we can say that:

a) The epidermis is presented as a hypoechoic line.

b) The dermis is presented as a hypoechoic band, shinier than the epidermis.

c) The subcutaneous tissue presents itself as a hypoechoic layer with the presence of longitudinal hypoechoic structures.

d) The echogenicity of each layer depends on its main component.
6 - Are characteristics of normal skin to ultrasound, except:

a) In the scalp, the hair follicles appear as hyperechoic structures which extend to the subcutaneous tissue.

b) In individuals with photodamage, a subepidermal band with low echogenicity is observed between the epidermis and the dermis.

c) In newborns, the echogenicity of the dermis is slightly hypoechoic.

d) In palmoplantar region, the epidermis presents with bilaminar aspect.

7 - Regarding the sonographic image of basal cell carcinoma, it is correct to state that:

a) Frequently the presence of hyperechoic points in its interior is observed, due to the presence of corneal cysts, microcalcifications and clusters of apoptotic cells within the tumor mass.

b) Generally it presents as hiperechogenic area contrasting with adjacent healthy tissue.

c) Its contour is always well defined and symmetrically distributed.

d) As in dermoscopy, it is possible to distinguish arboriform telangiectasia.

8 - Among the applications of HFUS in melanoma, we can highlight the following, except:

a) Establishing the tumor thickness.

b) Defining tumor margins.

c) Viewing of tumor vascularization.

d) Differentiating between melanoma and melanocytic nevus.

9 - Regarding the use of ultrasound for the detection of nodal metastases of melanoma, it is correct to state that:

a) It has a higher accuracy than the sentinel lymph node research.

b) It has better specificity and sensitivity than clinical examination.

c) Eliminates the use of RT-PCR during sentinel lymph node biopsy.

d) It has a higher sensitivity compared to scintigraphy.

10 - In ultrasound, which of the frequencies below are considered high:

a) $15-20 \mathrm{MHZ}$.

b) 3-6 MHz.

c) $10-18 \mathrm{MHz}$.

d) $6-13 \mathrm{MHz}$.

11 - Regarding the components of ultrasound equipment, it is correct to state that: 
a) The image obtained is best viewed in LCD screen.

b) In the two-dimensional ultrasound (B-mode), the brightness of each pixel corresponds to the frequency.

c) The transducer is the element that transforms electrical energy into mechanical acoustic wave and vice versa, and acts as transmitter and receiver.

d) The Doppler is based on the principle that sound waves emitted by the transducer and the reflector are static in relation to each other.

12 - Which of the components provides echogenicity to epidermis, dermis and subcutaneous tissue, respectively:

a) Lipid mantle, collagen, keratin.

b) Collagen, fat globules, keratin.

c) Keratin, collagen, fat globules.

d) Keratin, elastic fibers, collagen fibers.

13 - The ultrasound distinction between the basal and squamous cell carcinomas may be possible due to:

a) Difference in echogenicity.

b) Presence of hyperechoic points within the tumor mass.

c) Shrinkage of the collagen tissue.

d) Thickness and invasion to adjacent tissues.

\section{4 - Check the correct answer:}

a) In ultrasonographic analysis of melanocytic lesions, detection of blood vessels within the tumor mass shows $100 \%$ specificity and $34 \%$ sensitivity in distinguishing between benign and malignant lesions.

b) In basal cell carcinoma, intra and peritumoral blood flow is intense and consists of high flow arteries and veins.

c) In squamous cell carcinoma, the color Doppler shows a speckled pattern.

d) In melanoma, color Doppler demonstrates that vascularization is less intense than in benign lesions, predominating low flow arteries.

15 - Are factors that may lead to error in measurement of tumor thickness with HFUS, except:

a) Inflammatory process associated with neoplasia.

b) Procedures prior to the examination.

c) Presence of perilesional hypertrophic glands.

d) Large amount of intratumoral pigment.

16 - Regarding the ultrasonographic evaluation of skin lesions of systemic sclerosis, is it correct to state that:

a) Low echogenicity and increased skin thickness represents edema in the early stage of the disease.

b) The increase in skin thickness and echogenicity correspond to atrophy and fibrosis.

c) Increased echogenicity and decreased skin thickness represent edema in the early stage of disease

d) Differentiation between stages of the disease is not important in choosing the therapeutic approach.
17 - In HFUS, skin aging is characterized by:

a) Increased skin thickness.

b) Subepidermal band formation of low echogenicity.

c) Increased echogenicity of the dermis.

d) Increased hypoechoic images corresponding to the skin appendages in the dermis.

\section{8 - Regarding the use of the HFUS for monitoring of facial} fillers, it is incorrect to say that:

a) When located in the dermis, formation of hyperechoic structures is observed.

b) When located in the subcutaneous tissue, multiple anechoic pearls are observed.

c) When using filler composed of hyaluronic acid, ultrasound morphology depends on the injection plan.

d) This technique does not allow effective control of the site to be applied and the volume of the skin deposit.

19 - Among the various applications of HFUS, the following can be highlighted, except:

a) Pressure ulcer control.

b) Evolutionary monitoring of morphea clinical setting.

c) Distinction between benign and malignant melanocytic lesions.

d) Monitoring of plantar wart treatment.

20 - Ultrasonography is traditionally used in dermatology to investigate subcutaneous changes, such as nodules and infectious and inflammatory processes. High-frequency equipment present as differential features:

a) Visualization of superficial structures, allowing differentiation between skin layers.

b) Frequency from 6 to $15 \mathrm{MHz}$.

c) Low resolution for studying surface structures.

d) Color Doppler mapping allows differentiation of tumor cell lines.

\begin{tabular}{|c|c|c|c|}
\hline \multicolumn{4}{|c|}{$\begin{array}{l}\text { Answer key } \\
\text { Benign skin disease with pustules in the new- } \\
\text { born 2016;91(2):124-34. }\end{array}$} \\
\hline 1 - D & $6-A$ & $11-B$ & $16-C$ \\
\hline $2-C$ & $7-C$ & $12-C$ & $17-\mathrm{D}$ \\
\hline $3-\mathrm{A}$ & $8-C$ & $13-A$ & $18-\mathrm{A}$ \\
\hline 4 - D & 9 - B & $14-B$ & $19-D$ \\
\hline 5 - B & $10-D$ & 15 - D & $20-B$ \\
\hline
\end{tabular}

Papers

Information for all members: The EMC-D questionnaire is now available at the homepage of the Brazilian Annals of Dermatology: www.anaisdedermatologia.org.br. The deadline for completing the questionnaire is 30 days from the date of online publication. 